\title{
Android Malware Clustering through Malicious Payload Mining
}

\author{
Yuping $\mathrm{Li}^{1}$, Jiyong Jang ${ }^{2}$, $\mathrm{Xin} \mathrm{Hu}^{3}$, and Xinming $\mathrm{Ou}^{1}$ \\ 1 University of South Florida \\ yupingli@mail.usf.edu, xou@usf.edu \\ ${ }^{2}$ IBM Research \\ jjang@us.ibm.com \\ 3 Pinterest \\ huxinsmail@gmail.com
}

\begin{abstract}
Clustering has been well studied for desktop malware analysis as an effective triage method. Conventional similarity-based clustering techniques, however, cannot be immediately applied to Android malware analysis due to the excessive use of third-party libraries in Android application development and the widespread use of repackaging in malware development. We design and implement an Android malware clustering system through iterative mining of malicious payload and checking whether malware samples share the same version of malicious payload. Our system utilizes a hierarchical clustering technique and an efficient bit-vector format to represent Android apps. Experimental results demonstrate that our clustering approach achieves precision of 0.90 and recall of 0.75 for Android Genome malware dataset, and average precision of 0.98 and recall of 0.96 with respect to manually verified ground-truth.
\end{abstract}

\section{Introduction}

Triaging is an important step in malware analysis given the large number of samples received daily by security companies. Clustering, or grouping malware based on behavioral profiles is a widely-studied technique that allows analysts to focus their efforts on new types of malware. Multiple static [14, 30], dynamic [2, 22, and hybrid [12] analysis based clustering techniques have been proposed in the desktop malware domain.

With the rapid growth of Android smart devices, malicious Android apps have become a persistent problem. Security companies receive a list of (potential zero-day) malware on a daily basis [28]. Those apps that present certain suspicious behaviors but are not detected by any existing anti-virus scanners need to be further analyzed manually. Conducting clustering on those incoming malware apps can allow the analysts to triage their tasks by (a) quickly identifying malware that shares similar behaviors with known existing malware so they may not allocate much resources on it; and (b) selecting a few representative apps from each new malware cluster to prioritize their analysis. 
We often observe that existing approaches to group Android malware based on their behaviors have provided limited capabilities. For example, existing Android malware detection products may report a family name for a detected sample; however, samples from one family can have multiple different versions of malicious code segments presenting significantly different behaviors. Therefore, the malware family information provided by AV products can be incomplete to describe crucial malicious code segments of Android malware.

Existing overall similarity analysis based clustering system cannot be immediately applied for Android malware clustering because the malicious code segments often constitute only a small fraction of an Android malware sample. In desktop malware clustering, the static or dynamic features are first extracted from target samples. Then a clustering algorithm (e.g., hierarchical agglomerative clustering) is applied to group the samples such that samples within the same resulting group share high level of overall similarity. However, we note that overall similarity analysis performs poorly in Android malware clustering because of two common practices in Android malware development.

The first practice is repackaging. Malware writers may embed the malicious code inside an otherwise legitimate app, in which case the real malicious code segment is likely to be small compared to the original benign app. Our analysis shows that the ratio of the core malicious code segments to the entire app for a collection of 19,725 malware samples is between $0.1 \%$ and $58.2 \%$. Given the small percentage of malicious code segments, the conventional clustering approach that is based on overall code similarity will not work well. For example, two malicious samples from different families can be repackaged based on the same original benign app, thus presenting high level of overall similarity. Likewise, Android malware variants with the same malicious code of one family can be repackaged into different original benign apps, thus presenting low level of overall similarity.

Another practice is utilizing shared library code. Android apps often include a variety of third-party libraries to implement extra functionalities in a costeffective way. If the library code size is too large compared to the rest of the app, samples from different malware families may be clustered together simply because they share the same libraries. We measured the library code proportion of the 19,725 malware samples. For 13,233 of the samples that used at least one legitimate library, we found that the average library code ratio is $53.1 \%$ in terms of number of byte code instructions. This means a large portion of an Android app belongs to libraries. One approach to prevent those libraries from "diluting" the malicious code segments is to use a whitelist $4,6,8,10$ to exclude all library code. However, previous work leverages only the names of libraries while building a whitelist as opposed to the content of libraries. We observed that malware authors injected their malicious code under popular library names, such as com.google.ssearch, com. android.appupdate, android.ad.appoffer, and com. umeng. adutils. Consequently, naïve whitelisting approaches inadvertently remove certain malicious payloads together with the legitimate library code from analysis. We found that about 30\% of our analyzed Android malware families disguise their malicious payload under popular library names. 
Due to the above two reasons, directly applying overall similarity analysis on Android apps will not be effective for Android malware analysis. A major challenge is to precisely identify the malicious code segments of Android malware. For simplicity, we refer to the core malicious code segments of Android malware as malicious payload. A payload can be an added/modified part of a repackaged malware app, or the entire code of "standalone" malware app excluding legitimate library code.

In this paper we propose an Android malware clustering approach through iterative mining of malicious payloads. Our main contributions include:

1. We design and implement an Android malware clustering solution through checking if apps share the same version of the malicious payloads. By reconstructing the original malicious payloads, our approach offers an effective Android malware app clustering solution along with fundamental insights into malware grouping.

2. We design a novel method to precisely remove legitimate library code from Android apps, and still preserve the malicious payloads even if they are injected under popular library names.

3. We conduct extensive experiments to evaluate the consistency and robustness of our clustering solution. Our experimental results demonstrate that our clustering approach achieves precision of 0.90 and recall of 0.75 for Android Genome malware dataset, and average precision of 0.984 and recall of 0.959 regarding manually verified ground-truth.

\section{Overview of Android Malware Clustering System}

Rather than directly conducting overall similarity analysis between Android malware samples, we first design a solution to precisely remove legitimate library code from Android apps. We consider the shared code segments (excluding legitimate library code) between the analyzed Android apps as candidate payload, and find all of the input Android apps through pairwise intersection analysis. For a group of $n$ apps, each input app will contribute to $n-1$ versions of candidate payloads.

After extracting all candidate payloads, we conduct traditional clustering analysis on all candidate payloads to group similar ones together. Base on several key insights that are learned from analyzing candidate payload clustering results, we design an effective approach to iteratively mine the payload clusters that are most likely to be malicious, and make sure that each input app will only contribute one version of malicious payload. Finally, we use the identified malicious payload clusters and payload-to-app association information to group the input Android malware apps. We describe this process in more details below.

Figure 1 illustrates the overview of the clustering analysis system with five malware samples.

1. Library code removal: We convert malware samples into fingerprint representation, and design an effective approach to precisely remove legitimate 
Yuping Li, Jiyong Jang, Xin $\mathrm{Hu}$, and Xinming $\mathrm{Ou}$

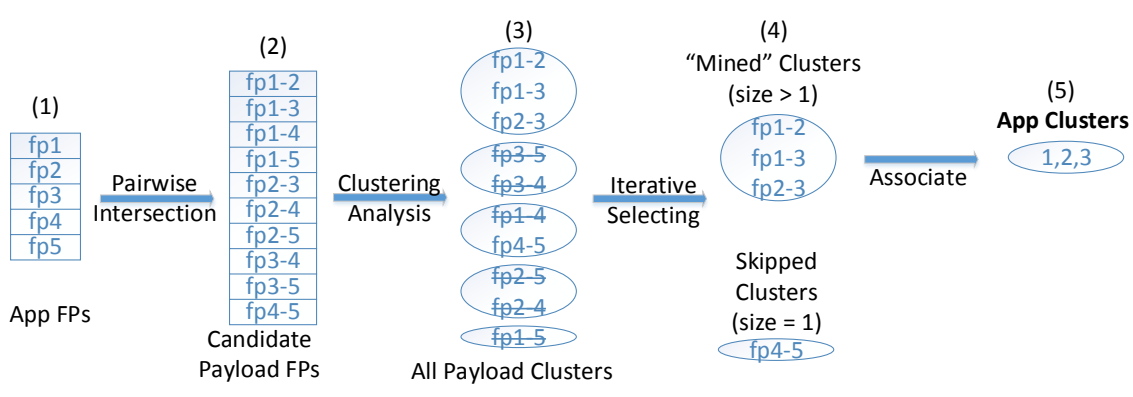

Fig. 1. Overview of the clustering system with five Android malware samples.

library code from each app fingerprint. We denote the library-removed app fingerprints as $\mathrm{fp} 1, \mathrm{fp} 2, \mathrm{fp} 3, \mathrm{fp} 4$, and $\mathrm{fp} 5$ accordingly.

2. Candidate payloads extraction: We conduct a pairwise intersection analysis to extract all shared code segments (e.g., candidate payloads) between input apps. Relying on the app fingerprint representation, we create candidate payload fingerprints, and record the payload-to-app association information. For example, fp1-2 indicates that this candidate payload is extracted from malware sample 1 and 2 .

3. Candidate payloads clustering: We then perform hierarchical clustering on all candidate payloads with a predefined clustering similarity threshold $\theta$, e.g., the candidate payload fingerprints $\mathrm{fp} 1-2, \mathrm{fp} 1-3$, and $\mathrm{fp} 2-3$ are grouped together as the largest payload cluster based on the overall payload similarity.

4. Malicious payload mining: After removing legitimate libraries, similar malicious payloads extracted from samples in the same malware family will become more popula ${ }^{\sqrt{4}}$ due to the "legitimate" reason of code reuse. Therefore, we design an iterative approach to mine the popular payload clusters from the clustering results, which are more likely malicious payload. For instance, candidate payload cluster containing fp1-2, fp1-3, and fp2-3 is selected as the most popular cluster. To ensure that each input app only contributes one version of final malicious payload, we simultaneously update the remaining payload clusters. e.g., fingerprints $\mathrm{fp} 1-4, \mathrm{fp} 1-5, \mathrm{fp} 2-4, \mathrm{fp} 2-5, \mathrm{fp} 3-4$, and fp3-5 are then skipped because malware sample 1, 2 and 3 have already been "used".

5. Malicious samples grouping: We group the original Android samples based on payload mining results and payload-to-app association information such that the samples within each app cluster contains the same version of the malicious payload. For example, malware samples 1, 2, and 3 are grouped together based on the selected candidate payload cluster containing fp1-2, fp1-3, and fp2-3.

\footnotetext{
${ }^{4}$ Further intuition explanation and popularity criteria are included in Section 4
} 


\section{App Fingerprint Representation and Utilization}

As we can see from Section 2, the clustering system requires an effective fingerprint representation to denote input Android apps and candidate payloads. Ideally, the fingerprint needs to be constructed from the code segments of the input app and support two fundamental operations: precisely removing legitimate code, correctly extracting shared app code.

Based on these requirements, we decide to represent Android apps as bitvector fingerprints, by encoding the features that are extracted from app code through feature hashing $13,14,26$. The value of each bit in the generated fingerprint is either 0 or 1, indicating whether the corresponding app has a specific feature or not.

This bit-vector format enables us to precisely remove legitimate library code (Section 3.2), extract shared code segments (Section 3.3), and reconstruct the original malicious payload (Section 3.4 by utilizing the bit manipulation capability.

\subsection{Fingerprint Generation and Fingerprint Comparison}

In this work, we use $n$-gram sequence of Dalvik bytecode to denote an Android app feature, and use a bit-vector fingerprint to represent the extracted features. The overall fingerprint generation process is shown in Figure 2.

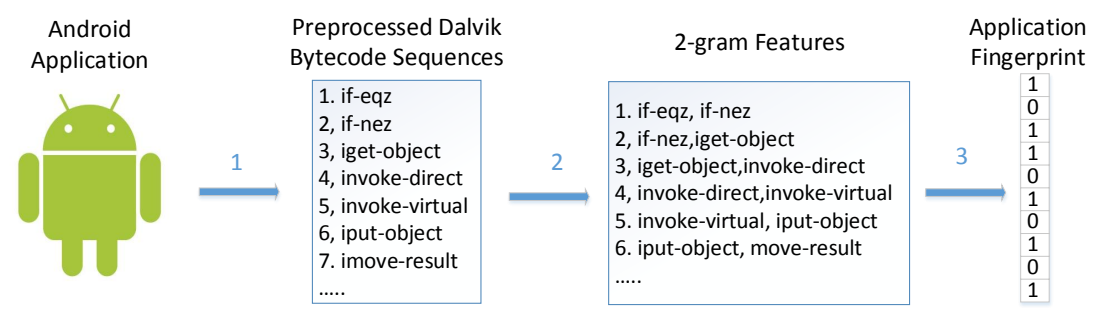

Fig. 2. Overall fingerprint generation procedure

For each Android app, we first use Dexdump 7 to disassemble classes .dex into Dalvik bytecode, then preprocess the Dalvik bytecode sequences to only include the major distinctive information and extract the $n$-gram features from the preprecessed bytecode sequences. We follow similar approach to extract the distinctive information (e.g., bytecode opcode) for feature construction as Juxtapp 11]. Since feature space is vital to support the key operations designed in this work, we decide to increase the feature space by including more generic but meaningful information from each bytecode instruction. The major distinctive information is separated into 4 categories and summarized in Table 1. Besides 
the feature differences shown in Table 1, we extract the $n$-gram features at the function level, while Juxtapp extracts $n$-gram features at the basic block level. For simplicity, we only show the Dalvik bytecode opcode sequences as the distinctive instruction information in Figure 2

Table 1. Major feature categories and main differences comparing with Juxtapp

\begin{tabular}{|l||c|c|c|}
\hline Feature Category & Examples & Our Approach & Juxtapp \\
\hline Dalvik bytecode opcode sequences & sget, goto, return & $\checkmark$ & $\checkmark$ \\
\hline Java VM type signatures & Z(Boolean), B(byte $)$ & $\checkmark$ & \\
\hline String value of const-string instructions & - & $\checkmark$ & \\
\hline Type signatures for "invoked" functions & f(I,[B)Z & $\checkmark$ & \\
\hline
\end{tabular}

After extracting all the $n$-gram features, we then encode all the features in a bit-vector format fingerprint through feature hashing technique using djb2 hash function. During feature hashing process, we use a tuple $A(i, j)$ to represent a feature position, in which $i$ is the function offset indicating from which function the particular $n$-gram feature is extracted, and $j$ is the bytecode offset indicating the position of the $n$-gram feature within the corresponding function. Then the feature-to-bit information is stored in a map, in which the key is the bit index within the fingerprint indicating where the feature is stored, and the value is the list of feature tuples that are mapped to the bit location. With increased feature space, we hope to reduce majority ofthe random feature collisions, and allow each bit index to represent the same $n$-gram feature content.

Similar to the complete Android apps, individual legitimate libraries and the candidate malicious payloads are also represented in the same size of bit-vector fingerprints. The concrete $n$-gram size and the fingerprint size used for clustering are determined through analyzing the collision rate of random features, which is discussed in Section 6.2.

To measure the similarity between two fingerprints, we use the Jaccard index, or the Jaccard similarity, which is defined as the size of intersection divided by the size of union of two sets. Since each fingerprint is a bit-vector, we leverage cache-efficient bit-wise AND $(\wedge)$ and bit-wise OR $(\vee)$ operations to compute the intersection and the union. Then, the similarity of two fingerprints $f p_{a}$ and $f p_{b}$ is defined as follows:

$$
\operatorname{Similarity}\left(\mathrm{fp}_{\mathrm{a}}, \mathrm{fp}_{\mathrm{b}}\right)=\frac{S\left(\mathrm{fp}_{\mathrm{a}} \wedge \mathrm{fp}_{\mathrm{b}}\right)}{S\left(\mathrm{fp}_{\mathrm{a}} \vee \mathrm{fp}_{\mathrm{b}}\right)},
$$

where $S(\cdot)$ denotes the number of 1-bits in the input.

Our fixed-sized bit-vector fingerprint representation also allows us to easily measure containment ratio in a similar fashion:

$$
\text { Containment }\left(\mathrm{fp}_{\mathrm{a}}, \mathrm{fp}_{\mathrm{b}}\right)=\frac{S\left(\mathrm{fp}_{\mathrm{a}} \wedge \mathrm{fp}_{\mathrm{b}}\right)}{S\left(\mathrm{fp}_{\mathrm{a}}\right)},
$$

which measures how much of the content of $\mathrm{fp}_{\mathrm{a}}$ is contained in $\mathrm{fp}_{\mathrm{b}}$. 


\subsection{Fingerprint based Library Code Removal}

To precisely remove legitimate library code without excluding a possibly injected malicious payload, we exclude legitimate library code from an app by removing the library-mapped bits from the app bit-vector fingerprint. For each legitimate library, we collect its official jar file and disassemble it into Dalvik bytecode sequences; then apply the same feature hashing technique to map the $n$-gram features of the library code into a bit-vector fingerprint $\mathrm{fp}_{\text {lib }}$. We then flip all the bits in the library fingerprint to get $\overline{\mathrm{fp}_{\mathrm{lib}}}$. Since the same features contained in an Android app and the library are mapped to the same bit positions in their fingerprint representation, we can exclude library-mapped bits from an app fingerprint by bit-wise ANDing $\overline{\mathrm{fp}_{\text {lib }}}$ and $\mathrm{fp}_{\mathrm{app}}$. Figure 3 demonstrates the overall procedure to safely remove legitimate twitter $4 \mathrm{j}$ library code from a malware sample.

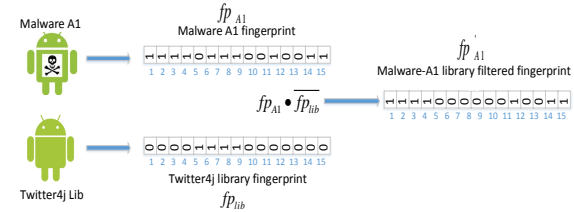

Fig. 3. Example procedure to safely remove legitimate "twitter4j" library code

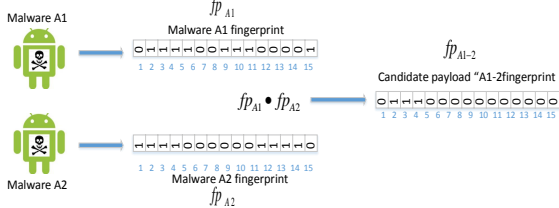

Fig. 4. Extracting a candidate payload from two malware applications

We first conduct statistical analysis for the disassembled apps to identify the embedded legitimate libraries, and record the years when the target samples were created. We then obtain 5 the officially released library jar files to create the corresponding library fingerprints, and remove the library code from the analyzed apps. The library code removal process is applied only when an app contains code snippets that are defined under corresponding library namespaces.

In our implementation, each library is represented with an individual fingerprint. We encode multiple versions of the same library together in a single library fingerprint. This aggregated library representation may cause potential feature collision between the app code and the irrelevant versions of the library code. However, we empirically demonstrate in Section 6.3 that the library code removal process is precise because different versions of the same library typically share high level of code similarity due to code reuse, and the size of the single library is often smaller than the entire app.

\footnotetext{
${ }^{5}$ We randomly select one version of library in each year in case there are multiple versions of libraries released within the same year.
} 


\subsection{Fingerprint based Candidate Payload Extraction}

The next operation is to extract malicious payloads from malware samples. We consider the shared code segments (after excluding legitimate libraries) between each malware sample pair to be a candidate malicious payload. For a group of malware samples, we obtain the intersection of every fingerprint pair of libraryexcluded samples, and consider the shared 1-bits between the sample fingerprints as a candidate payload fingerprint.

Figure 4 describes the intersection analysis procedure to extract a candidate malicious payload at a high level. For two malware samples we first build their fingerprints and exclude the legitimate library bits from the fingerprints. Then we pinpoint their shared 1-bits (e.g., bits index 2, 3, and 4) as potentially malicious ${ }^{6}$ bits and construct a candidate payload fingerprint.

During the candidate payload extraction process, we keep track of the association information between the candidate payload (e.g., A1-2) and the corresponding samples (e.g., A1 and A2). We subsequently use the payload-to-app association information and the malicious payload mining results to group malware samples.

\subsection{Fingerprint based Malicious Payload Reconstruction}

Using the bit-vector fingerprint representation, we can also define the cluster fingerprint for a version of the candidate payload cluster as the intersection of all the candidate payload fingerprints in the cluster. The 1-bits contained in the resulting cluster fingerprint can be viewed as the shared malicious bits for all input apps that share the same version of malicious payload.

Using the identified malicious bits from app fingerprints, we can then reconstruct the corresponding malicious payload code by checking the feature-to-bit mapping information that was recorded during feature hashing, which can be viewed as the reverse procedure of fingerprint generation. Given the identified malicious bits, we locate the feature tuples that are mapped to those identified malicious bits. We use each retrieved feature tuple to locate the $n$ lines of code where the $n$-gram feature is extracted, then reconstruct complete malicious code sequences by properly stitching the identified $n$ lines of code segments together.

In practice, feature collision is possible but becomes negligible with appropriate $n$-gram size and fingerprint size, thus we will rarely recover the irrelevant code. To certain extent, payload code reconstruction compensates feature hashing collisions (e.g., resulting in missing $n$-grams) as far as the missing $n$-gram is within the overlapped original code sequences of recovered features. The reconstructed malicious payload code can be further inspected to verify its maliciousness.

\footnotetext{
${ }^{6}$ malicious payload mapped
} 


\section{Malicious Payload Mining}

Key insights: (a) In practice, when feature hashing is configured to have a low collision rate, malware app fingerprints will not contain a large number of shared 1-bits unless they do share certain common features (e.g., payload code snippets). (b) Likewise, if a target dataset contains malware samples that do share the same version of the malicious payload, then the candidate payload fingerprints extracted from those samples will contain similar shared 1-bits and be automatically clustered into the same group. (c) After removing legitimate library code from an app, similar malicious payloads have higher chances to form a larger cluster than the ones related to less popular libraries or coincidentally shared code segments. (d) Compared to coincidentally shared code segments, similar malicious payloads will have a larger shared code base because of "legitimate" reason of code reuse in the same malware family, and the fingerprints for the malicious payloads will have a larger amount of shared 1-bits.

Based on the above key insights, we design the following strategies to iteratively select representative candidate payload clusters based on payload popularity, which is determined based on the three criteria: the entry size of a payload cluster $l$, the number of distinct apps associated with a payload cluster $m$, and 1-bits count of a payload cluster fingerprint $k$.

- We count the number of candidate payload fingerprint entries in each cluster, and maximize the possibility of extracting core malicious payloads by selecting the clusters with the largest number of payload fingerprint entries. Payload cluster size $l$ is a direct indicator for the popularity of the shared code segments between malware samples, and such popular shared code is a good candidate for one version of malicious payloads since we have already filtered out popular legitimate library code.

- We measure the distinct apps $m$ that contribute to generating candidate payload fingerprints of each cluster, and select the clusters with the largest number of distinct apps if they have the same number of payload entries. Payload clusters that contain a large number of unique payload entries are often associated with a large number of distinct apps, and we use this app association information to break the tie in case the number of cluster entries are the same since distinct apps can be considered as another sign of comparative popularity.

- We obtain the intersection bits $k$ of payload fingerprint entries in each cluster as the cluster fingerprint. If two clusters are associated with the same number of distinct apps, we then select the one with the larger number of 1-bits in its cluster fingerprint. In this way, we can extract the payload with a larger code size, and it helps to increase the likelihood of getting malicious payloads together with shared libraries, and we subsequently exclude possibly remaining libraries later.

- During cluster selection, we keep track of which apps have been used to generate candidate payload fingerprints in the previously selected clusters, and consider already-selected apps as "inactive". We update the remaining 
Yuping Li, Jiyong Jang, Xin Hu, and Xinming Ou

payload clusters by removing candidate fingerprint entries that are associated with "inactive" apps. Skipping such fingerprints allows us to extract one version of the malicious payload from each app. This helps to merge all the shared core malicious code together, and only extract the widely shared malicious code between all apps, which also helps to reduce the probability of extracting non-malicious payload code.

- We omit a payload cluster if the corresponding cluster fingerprint contains less than the minimum $k$ number of 1-bits, meaning that the extracted code segments are too small. It forces the algorithm to break the current large payload cluster into smaller clusters with a larger code size, and prevent different malware families from being clustered together. We set the minimum number of 1-bits $k$ to 70 since the majority of the analyzed Android malware app fingerprints had more than 70 1-bits.

- We exclude a candidate payload cluster if it becomes empty after the update in the last step, or if the number of payload fingerprint entries is too small $(e . g ., l=1)$. This is because Clusters with only a single candidate payload entry provide little additional popularity information, and are more likely to contain less popular libraries or other coincidentally shared code snippets. We consider malware samples associated with such payload clusters as unclustered, and the unclustered app is evaluated as a singleton.

The shared payloads between Android samples can be library code segments, malicious payloads, copy-and-pasted code segments, or other coincidentally shared code segments. The above payload mining strategy enables us to select the most likely malicious candidate payload groups. Legitimate non-library reused code may be collected together with malicious payload only if it is shared across a significant number of apps. Otherwise, the less popular legitimate nonlibrary code will be evidently excluded during the (popularity-based) payload mining procedure. If the same benign app is indeed used by many malware apps, we can further exclude original benign app code (i.e., the legitimate non-library reused code) in a similar way to remove library code using a benign app fingerprint.

\section{Optimize Overall Clustering Efficiency}

According to the previously discussed malicious payload mining procedure, we will generate $\frac{n \times(n-1)}{2}$ versions of candidate payload fingerprints given $n$ malware samples, but the hierarchical clustering algorithm also has a quadratic complexity with respect to the number of analyzing targets. Due to the overall quartic complexity of the algorithm, directly using it to analyze large number of samples becomes a time-consuming task. Therefore, we further develop two methods to improve the scalability of the clustering analysis procedure, and hereafter refer them as Opt-1, and Opt-2. 


\subsection{Opt-1: Optimize Each Pairwise Computation}

The first method to speed up the overall clustering process is to optimize each pairwise computation. Broder proposed minHash $[3$ to quickly estimate the Jaccard similarity of two sets without explicitly computing the intersection and the union of two sets. By considering our bit-vector fingerprint as a set, we apply minHash to further transform a large fingerprint into a smaller size signature, and calculate the similarity of minHash signatures to estimate the Jaccard similarity of the original fingerprints.

To apply minHash, we define a minHash function output of our bit-vector fingerprint $h(\mathrm{fp})$ to be the first non-zero bit index on a randomly permutated bits order of the fingerprint. We then apply the same minHash function to two fingerprint $\mathrm{fp}_{\mathrm{a}}$ and $\mathrm{fp}_{\mathrm{b}}$. This will generate the same minHash value when the first non-zero bit indexes for two fingerprints $f p_{a}$ and $f p_{b}$ are the same. Since the probability that the firstly encountered bit is a non-zero bit for $\mathrm{fp}_{\mathrm{a}}$ and $\mathrm{fp}_{\mathrm{b}}$ is conceptually the same as Jaccard similarity Similarity $\left(\mathrm{fp}_{\mathrm{a}}, \mathrm{fp}_{\mathrm{b}}\right)[18$, we use such probability $\operatorname{Pr}\left[h\left(\mathrm{fp}_{\mathrm{a}}\right)=h\left(\mathrm{fp}_{\mathrm{b}}\right)\right.$ to estimate the original Jaccard similarity.

The probability estimation becomes more accurate if more independent minHash functions are used together. Formally, we define a minHash signature $\operatorname{sig}(\mathrm{fp})$ to be a set of $k$ minHash function values extracted from $k$ round of random permutations over the fingerprint, and represent it as follows: $\operatorname{sig}(\mathrm{fp})=$ $\left[h_{1}(\mathrm{fp}), h_{2}(\mathrm{fp}), \ldots, h_{k}(\mathrm{fp})\right]$. We denote the similarity of two minHash signatures as the ratio of equal elements between $\operatorname{sig}\left(\mathrm{fp}_{\mathrm{a}}\right)$ and $\operatorname{sig}\left(\mathrm{fp}_{\mathrm{b}}\right)$.

Instead of maintaining $k$ random permutations over the bit-vector, we follow a common practice for using minHash technique and use $k$ different hash functions to simulate $k$ random permutations, where each hash function maps a bit index to a value. In order to create $k$ hash functions, we first generate $k$ random numbers, then use FNV 9] hash algorithm to produce a basic hash output for each bit index, and finally apply XOR operation between each random number and the hash output to get the $k$ hash outputs. For each hash function, we select the smallest hash value (to simulate the first non-zero bit index) over all of the bit indexes of the fingerprint as the final hash output.

Note that the FNV hash value and the $k$ random numbers are all 32 bits unsigned integers, and they can be used to safely simulate random permutation over $512 \mathrm{MB}$ bit-vector fingerprint. In practice, the $k$ value usually needs to be larger than 100 to generate good enough results 18 . We set $k$ to be 256 in our experiments, and thus convert each bit-vector fingerprint into a $1 \mathrm{~KB}$ minHash signature.

In order to evaluate the potential impact of Opt-1 on accuracy, we conduct two experiments on the smallest 50 malware families: 7 ; one experiment (Exp-1) with no optimization, and another experiment (Exp-2) using Opt-1. We used the clustering output from Exp-1 as a reference, and measured the precision and recall of the clustering output from Exp-2. The precision and recall indicate how

\footnotetext{
${ }^{7}$ We select those families since their maximum family size is under 100 and all the experiments for those families can be finished within 1 hour.
} 
Yuping Li, Jiyong Jang, Xin Hu, and Xinming Ou

similar the two experiments results are, and are used to check the impact on accuracy brought by Opt-1.

Our experiments showed that on average Exp-2 took less than $83 \%$ time to complete compared to Exp-1 for the analyzed families, and the average precision and recall of the clustering output were 0.993 and 0.986 . Opt- 1 significantly reduce the overall memory consumption with minHash signature representation and improve the pairwise computation efficiency with almost zero accuracy penalty.

\section{$5.2 \quad$ Opt-2: Employ approximate clustering}

The previous optimization is still not sufficient for using the algorithm to analyze large scale malware samples. For instance, when analyzing with 2,000 samples, the algorithm will create 1,999,000 candidate payloads, and it results in approximately $2.0 \times 10^{12}$ pairwise comparison. Even $1 \%$ of the total comparison still takes lots of computation resources. To resolve the scalability issue for a large dataset input, we further adopt prototype-based clustering technique [16 22 2 to achieve approximate clustering.

Specifically, we randomly divide the target samples into small size (e.g., 150) groups. For each group, we apply hierarchical clustering analysis on the shared payload within the group, and create a prototype fingerprint for each payload cluster by applying intersection analysis (to obtain all the shared 1-bit) among the payload fingerprints in each cluster. We then conduct hierarchical clustering analysis on all the collected prototype fingerprints. In this way, we represent a group of similar payload fingerprints with a single prototype fingerprint, and the algorithm proceeds with approximate clustering analysis using the prototype fingerprints instead of the original payload fingerprints.

We design two experiments to evaluate the impact of Opt-2 on accuracy: one experiment (Exp-3) using Opt-1 only, and another experiment (Exp-4) using Opt-1 and Opt-2. Due to the quartic complexity of the original algorithm, the overall analysis (using Opt-1 only) will get dramatically slower for analyzing larger number of malware samples. For instance, we found it takes about one day to analyze 1000 samples and more than five days to analyze 2000 samples for Exp-3. In order to conduct the evaluation within reasonable amount of time, we randomly select $70 \%$ of labeled samples from the largest 4 malware families and conduct the two experiments for each family. We used the clustering output generated by Exp-3 as reference, and measured the precision and recall of the clustering output generated by Exp-4 to evaluate the accuracy impact brought by Opt-2.

Our experiments showed that on average Exp-4 can speed up more than 95\% compared to Exp-3, and the average precision and recall for the analyzed 4 families were 0.955 and 0.932 . This optimization makes it feasible to apply our algorithm to analyze a bigger scale of malware families while providing a desirable trade-off option between speed and accuracy. 


\section{$6 \quad$ Experiments}

In this section, we describe the data preparation procedure, and report malware clustering results and key findings of our experiments.

\subsection{Data Preparation}

We obtained a large collection of potentially malicious Android apps (ranging from late 2010 to early 2016) from various sources, include Google Play, VirusShare 23] and third party security companies. In order to prepare groundtruth family labeling for the datasets, we queried the collected apps against VirusTotal 29] around April 2016, and used the scanning results to filter out potentially ambiguous apps.

To assign family labels to the collected malware samples, we applied the following steps: (1) tokenized VirusTotal scanning results and normalized the contained keywords, and then counted the total number of occurrences of each keyword. (2) removed all the generic keywords such as Virus, Trojan, and Malicious. (3) detected keyword aliases by calculating the edit distances between keywords. For example, Nickyspy, Nickspy, Nicky, and Nickibot were all consolidated into Nickispy. (4) assigned the dominant keyword as the family label for the sample. A keyword was considered as dominant if it satisfied two conditions: (a) the count of the keyword was larger than a predefined threshold $t(e . g ., t=10)$, and (b) the count of the most popular keyword was at least twice larger than the counts of any other keywords.

Table 2. Clearly Labeled Malware Families

\begin{tabular}{|c|c||c|c||c|c||c||c|c|c|}
\hline Name & Size & Name & Size & Name & Size & Name & Size & Name & Size \\
\hline Dowgin & 3280 & Minimob & 145 & Erop & 48 & Vidro & 23 & Koomer & 15 \\
Fakeinst & 3138 & Gumen & 145 & Andup & 48 & Winge & 19 & Vmvol & 13 \\
Adwo & 2702 & Basebridge & 144 & Boxer & 44 & Penetho & 19 & Opfake & 13 \\
Plankton & 1725 & Gingermaster & 122 & Ksapp & 39 & Mobiletx & 19 & Uuserv & 12 \\
Wapsx & 1657 & Appquanta & 93 & Yzhc & 37 & Moavt & 19 & Svpeng & 12 \\
Mecor & 1604 & Geinimi & 86 & Mtk & 35 & Tekwon & 18 & Steek & 12 \\
Kuguo & 1167 & Mobidash & 83 & Adflex & 32 & Jsmshider & 18 & Spybubble & 12 \\
Youmi & 790 & Kyview & 80 & Fakeplayer & 31 & Cova & 17 & Nickispy & 12 \\
Droidkungfu & 561 & Pjapps & 75 & Adrd & 30 & Badao & 17 & Fakeangry & 12 \\
Mseg & 245 & Bankun & 70 & Zitmo & 29 & Spambot & 16 & Utchi & 11 \\
Boqx & 214 & Nandrobox & 65 & Viser & 26 & Fjcon & 16 & Lien & 11 \\
Airpush & 183 & Clicker & 58 & Fakedoc & 26 & Faketimer & 16 & Ramnit & 9 \\
Smskey & 166 & Golddream & 54 & Stealer & 25 & Bgserv & 16 & & \\
Kmin & 158 & Androrat & 49 & Updtkiller & 24 & Mmarketpay & 15 & & \\
\hline
\end{tabular}

Although our malware labeling process may look similar to AVclass 27], we developed the approach independently without the knowledge of the AVclass; and both work was finished around the same time. The unlabeled samples were 
Yuping Li, Jiyong Jang, Xin Hu, and Xinming Ou

not included in the malware dataset for clustering analysis. In summary, we collected 19,725 labeled malware samples from 68 different families, and the detailed breakup of the malware samples is shown in Table 2

Besides the above labeled malware dataset, we also collected Android Genome malware samples 34 to obtain an optimal clustering threshold, and randomly selected a list of 10,000 benign samples from AndroZoo [1 to evaluate the accuracy of the library removal procedure. In particular, we selected benign apps that were created around the same time (before Jan 1st, 2016) as most of the labeled malware samples, and their latest (Mar 2017) VirusTotal re-scanning results showed no malicious labels.

\subsection{Feature Collision Analysis}

The accuracy of the proposed clustering system and the correctness of the reconstructed malicious payloads relies on the assumption that unique features will be mapped to unique bit locations within the bit-vector fingerprint. Feature collision is directly impacted by two parameters: an $n$-gram size, and a bit-vector fingerprint size. To evaluate a feature collision rate, we varied the $n$ gram size (2 and 4) and the bit-vector fingerprint size, and then measured how many unique features were mapped to the same single bit position, i.e., feature collision. Figure 5 illustrates feature collision with regard to different $n$-gram sizes and fingerprint sizes.

The graph shows that feature collision occurs more frequently when the fingerprint size is small. The total number of unique features depends on the $n$-gram size. For the labeled malware, it was about 4.1 million for 2-gram features, and 14.4 million for 4-gram features. And for the benign dataset, it was about 15.2 million for 2-gram features, and 45.3 million for 4-gram features. According to the pigeonhole principle, when putting $\mathrm{N}$ unique features into $\mathrm{M}$ buckets, with $N>M$, at least one bucket would contain more than one unique features. This means that we need to set the bit-vector fingerprint size larger than the total number of unique features to reduce feature collision. Therefore, we set the default $n$-gram size to be 2 and default fingerprint size to be $1024 \mathrm{~KB}$ which provides $8,388,608$ unique bit positions. With the above configuration, the unique feature per bit value was reduced to 0.49 to process the labeled malware dataset. Notice that the complete feature space is unlimited for our system due to the inclusion of arbitrary string values, however the true unique features contained in a certain dataset will be limited.

\subsection{Library Removal Accuracy}

Besides the random feature collision discussed in the previous section, it is also possible that feature collision may happen between the app code and the irrelevant versions of the library code. To evaluate the library removal accuracy, we assumed the libraries used in benign samples were not purposefully manipulated, and measured the precision (e.g., how much of the removed code is true library code) and recall (e.g., how much of the true library code is removed) of 


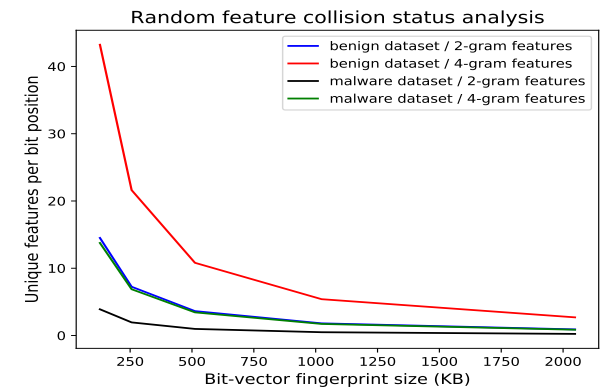

Fig. 5. Random feature collision status

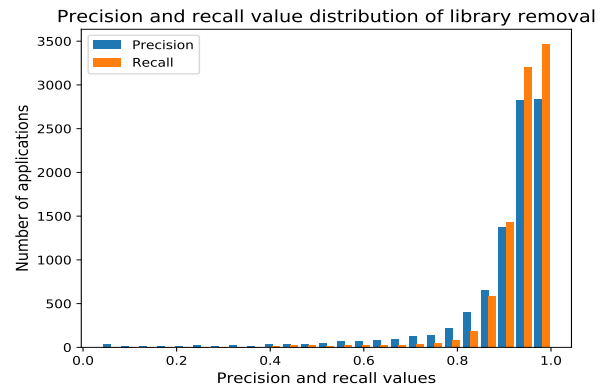

Fig. 6. Benign apps lib removal accuracy

library code removal results for the prepared benign samples. Particularly, we considered the code that were defined under the official library names in the benign samples as ground truth library code, and created the true library code fingerprint $\mathrm{fp}_{\text {true }}$ by mapping all the features from the true library code to a bit-vector fingerprint. After removing the library code from each app, we identified the bit positions that were presented in the original app fingerprint and were removed subsequently; and used the identified bit positions to generate removed library code fingerprint $\mathrm{fp}_{\text {removed }}$. Using the containment ratio calculation function as discussed in Section 3.1, library removal precision $\mathrm{P}_{\text {lib }}$ is defined as $\frac{S\left(\mathrm{fp}_{\text {true }} \wedge \mathrm{fp}_{\text {removed }}\right)}{S\left(\mathrm{fp}_{\text {removed }}\right)}$, and library removal recall $\mathrm{R}_{\text {lib }}$ is defined as $\frac{S\left(\mathrm{fp}_{\text {true }} \wedge \mathrm{fp}_{\text {removed }}\right)}{S\left(\mathrm{fp}_{\text {true }}\right)}$, where $S(\cdot)$ denotes the number of 1-bits in the bit-vector.

Figure 6 depicts the library removal precision and recall for the benign apps. We observed that 9,215 benign apps contained at least one legitimate library, and the median values for precision and recall were $0.94,0.95$, respectively. We manually inspected certain corner cases with poor precision or recall. The poor precision cases were due to incomplete true library code extraction, e.g., an older version of Admob library contained obfuscated version of code which were not under com.google domain, thus not counted as true library code. The poor recall cases were due to excessive true library code inclusion, e.g., all the code of the Androidify app was defined under com.google domain which made the distinction of library code obscure.

\subsection{Malware Clustering Results}

In order to select an optimal clustering threshold for the system and assess the performance comparing with other known Android malware clustering system, we first applied our clustering system on the Android Genome malware dataset. We used the classical precision and recall [2, 12, 14, 19, 22, 30] measurements to evaluate the accuracy of clustering results. Figure 7 describes the clustering precision and recall results with various thresholds.

The highest F-measure score was 0.82 with precision of 0.90 and recall of 0.75 when the clustering threshold was 0.85 . We set the default clustering threshold 
value to be 0.85 for subsequent clustering analysis. As a reference, ClusTheDroid 17 achieved precision of 0.74 and recall of 0.73 while clustering 939 of Android Genome malware samples.

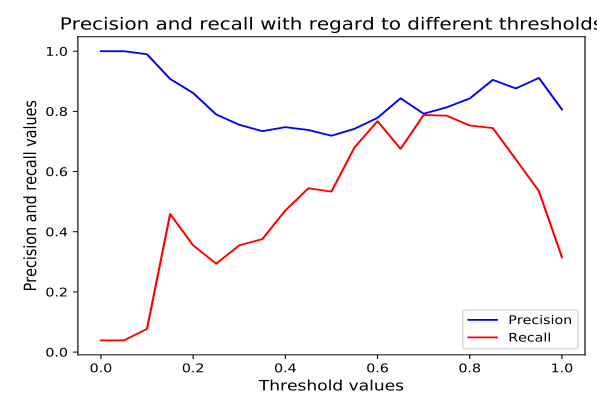

Fig. 7. Clustering results of Android Genome malware dataset

\begin{tabular}{|c||c|c|c|c|}
\hline Datasets & Samples & Clusters & Precision & Recall \\
\hline \hline D1 & 1064 & 33 & 0.977 & 0.972 \\
\hline D2 & 1462 & 27 & 0.987 & 0.964 \\
\hline D3 & 1708 & 29 & 0.985 & 0.978 \\
\hline D4 & 1039 & 31 & 0.971 & 0.960 \\
\hline D5 & 2277 & 29 & 0.988 & 0.989 \\
\hline D6 & 1066 & 30 & 0.971 & 0.919 \\
\hline D7 & 1256 & 29 & 0.985 & 0.981 \\
\hline D8 & 1680 & 29 & 0.985 & 0.980 \\
\hline D9 & 2074 & 31 & 0.996 & 0.858 \\
\hline D10 & 1612 & 31 & 0.992 & 0.989 \\
\hline
\end{tabular}

Fig. 8. Clustering results of different subversion datasets

Note that the clustering outputs produced by our system is per sub-version instead of per family, therefore it is more challenging to properly obtain finegrained ground truth labels to evaluate the accuracy. In fact, this was the main reason for a bit low recall of our system with respect to coarse-grained ground truth labels, e.g., one Android malware family samples might contain multiple versions of malicious payloads. While reviewing the clustering results, we noticed that 13 families of the Genome dataset contained more than one versions of malicious payloads. For example, Basebridge contained 7 versions of malicious payloads with threshold of 0.85 .

Therefore, we separated the labeled malware samples into sub-versions using the clustering system, and further designed several experiments to evaluate the clustering results with manually verified sub-version ground-truth. We manually verified the correctness of the sub-version cluster results. For the generated sub-version clusters, we first checked if the extracted payload was the indeed malicious. Since each version of the extracted payloads usually had similar class names and Dalvik code sequences, the maliciousness of the extracted payload can be spotted by checking the extracted class names (e.g., similar pseudo-random pattern). In case the class names were not enough to determine its maliciousness, we then went through the reconstructed code segments and checked if there were any suspicious activities or behaviors, such as stealthily sending out premium SMS. After verifying the maliciousness of the extracted payload, we then randomly selected 3 samples from each sub-version group, and checked if the selected apps contained the same version malicious payload. Out of 19,725 malware samples that were labeled with 68 families, we obtained a total of 260 verified sub-version clusters, and each cluster corresponded to one version of the malicious payloads. 
We considered the VirusTotal family labels together with the manually verified sub-version information as ground truth, and prepared 10 experiment datasets. For each dataset, we randomly selected 30 sub-versions from the entire ground truth dataset (e.g., 260 sub-versions), then mixed the selected samples together as one input dataset. The resulting datasets had different overall sizes as each individual sub-version had different numbers of samples. The detailed dataset sizes and sample clustering results for the 10 datasets are presented in Figure 8 . On average, the sample clustering algorithm separated the input malware samples into 29.9 clusters, which was extremely close to the reference set (i.e., 30 sub-versions). For the 10 experiment datasets, the clustering algorithm achieved average precision of 0.984 and average recall of 0.959 , the worst precision and recall for clustering multiple malware families were 0.971 and 0.858 , which suggests that the clustering system generated consistent and reliable outputs.

\subsection{Key Findings for Malicious Payload Analysis}

In this section, we report the key findings learned from the malware sub-version verification process.

Significant library code ratio: From the labeled malware datasets, we found that the average library code ratio was larger than $50 \%$ for the malware samples that contained at least one legitimate library. This highlights that existing Android malware similarity analysis work becomes ineffective without properly handling library code.

Limited versions of malicious payloads: During our experiments, we acquired 260 versions of malicious payloads from 68 labeled malware families while conducting clustering of each family. Among the 68 malware families, 27 families had only one version of malicious payload, and 5 families had more than 10 different versions of malicious payloads. For example, Dowgin was the largest malware family and had 23 versions of malicious payloads extracted.

Malicious payload under popular namespaces: We conducted manual analysis on the extracted malicious payloads, and noted that $29 \%$ of Android malware families injected their malicious payloads under popular namespaces, such as com.google and com. android, or legitimate advertisement library namespaces like com. umeng. Table 3 in Appendix includes the detailed malicious payload findings for the identified families. Since com.google and com.android are the main class names used by Android Open Source Project and Google Mobile Services, such malicious payloads can easily get overlooked.

\section{Limitation}

Our Android malware clustering approach is based on the assumption that malware authors often reuse the same malicious payload to create new malicious samples, and the obfuscated code sequences of malicious payload would largely remain the same if they are generated by the same obfuscation tool. This is consistent with our findings as listed in Section 6.5. Theoretically, advanced 
Yuping Li, Jiyong Jang, Xin Hu, and Xinming Ou

obfuscation techniques (e.g., class encryption or dynamic loading) can eventually break the assumption by generating a new version of a malicious payload for every new malware instance, or completely removing the original malicious payload from classes.dex. The attack and defense against malware obfuscation is a long-term arms race, and has already been observed in the traditional desktop malware analysis domain. For example, as observed in desktop malware research 152024 , independent systems might be desirable to specifically handle the de-obfuscation process. We consider it as a separate pre-processing step for malware analysis, and leave a comprehensive solution for advanced obfuscation as an orthogonal problem. In addition, using dynamic analysis with a sandbox can help further analyze malware. However, dynamic analysis also suffers from its own limitations, such as sandbox evasion and code coverage.

We believe that the Android malware analysis community can benefit from our work in several aspects. (a) It offers an alternative malicious payload extraction approach in which we can extract a more complete version of malicious payloads even if the malicious payloads are injected under popular library names or under existing functions. (b) It provides a viable solution for conducting Android malware clustering analysis by checking if malware samples contain the same version of malicious payloads. (c) Majority of Android malware samples are not obfuscated or obfuscated by simple obfuscation tools, even for the samples we collected recently. For example, within the extracted 260 versions of malicious payloads, we observed 181 of them had plain code, and only 79 of them used naming obfuscation, which was a simple basic obfuscation technique being used in practice. (d) As long as there are shared malicious code segments regardless of obfuscation among the samples from the same malware family, our algorithm extracts the shared patterns and uses them for deciding malware clustering output.

\section{Related Work}

\subsection{Android Malware Clustering and App Similarity Analysis}

Due to the challenges that are discussed in Section 1, existing Android malware clustering approaches have not been widely adopted yet. ClusTheDroid [17] was a system for clustering Android malware using 38 features extracted from profiles of reconstructed dynamic behaviors. Samra 25 extracted features from Android app manifest files, and could only cluster applications into two categories using K-means algorithm. Without properly excluding the features or behaviors that belong to the original benign apps or legitimate libraries, traditional clustering approaches would not be able to produce promising results.

Similarity analysis is essential for clustering, but existing Android application similarity analysis techniques were mainly designed to detect repackaged apps 11, 31, 33], and such overall similarity analysis based techniques cannot be directly applied for Android malware clustering for reasons described in Section 1. SMART 21] proposed a semantic model for Android malware analysis, but was mainly built for malware detection and classification. Both Juxtapp 11. 
and our system use $n$-gram bytecode features and feature hashing [13, 14,26$]$ as basic building blocks. However, Juxtapp excluded library code for further analysis if the core application component does not directly invoke it, which still couldn't differentiate a legitimate library and a bogus library with the same legitimate name. Furthermore, directly using Juxtapp to cluster Android malware will suffer the same limitations like other traditional clustering methods as it is based on overall similarity.

\subsection{Android Malicious Payload Analysis}

Malicious payload identification and extraction is essential for Android malware analysis. Zhou and Jiang [34] manually analyzed malicious payloads of Android malware and summarized the findings in the Android Malware Genome project. DroidAnalytics 32 presented a multi-level signature based analytics system to examine and associate repackaged Android malware. MassVet [5] analyzed graph similarity at the function level and extracted the shared non-legitimate functions as malicious payloads through commonality and differential analysis, and it applied a whitelist to exclude legitimate library code from analysis.

MassVet [5] is close to our work in that both extract malicious payloads from Android malware. However, similar to existing Android malware analysis work [4 6, 8, 10, MassVet simply used library name based whitelists to exclude popular library code, which can result in the failure of malicious payload extraction, and lead to false negatives in malware detection if malicious payloads are injected under popular library namespaces. In addition, due to the function level payload granularity of MassVet, it can not be easily designed to achieve payload-sharing based Android malware clustering, since the same function could be shared by different malware families, and the malware samples from the same family usually share multiple functions at the same time. Last but not least, MassVet won't be able to extract malicious payload injected under existing functions, while the instruction level payload granularity designed by our approach enables us to precisely identify one version of malicious payload from each Android malware, which includes all of the malicious components even if they are injected in existing functions or across different functions.

\section{Conclusion}

In this paper, we proposed a practical solution to conduct Android malware clustering. As an internal component, the fingerprint based library removal technique was used to distinguish a legitimate library and a bogus library that may share the same library name. Unlike traditional clustering techniques which examine the overall similarity, we achieved Android malware clustering by checking whether the analyzed Android malware samples shared the same version of malicious payload code. Compared with existing malicious payload extraction system, our approach extracts malicious payloads even if they were injected under popular library namespaces or under existing benign functions, and it provides 
Yuping Li, Jiyong Jang, Xin Hu, and Xinming Ou

a more complete picture of the whole malicious payload. Our comprehensive experimental results demonstrate that our clustering approach generates consistent and reliable outputs with high precision and recall.

\section{Acknowledgment}

This work was partially supported by the U.S. National Science Foundation under Grant No. 1314925 and 1622402. Any opinions, findings and conclusions or recommendations expressed in this material are those of the authors and do not necessarily reflect the views of the National Science Foundation.

\section{References}

1. Allix, K., Bissyandé, T.F., Klein, J., Le Traon, Y.: Androzoo: Collecting millions of android apps for the research community. In: Proceedings of the 13th International Conference on Mining Software Repositories. pp. 468-471. ACM (2016)

2. Bayer, U., Comparetti, P.M., Hlauschek, C., Kruegel, C., Kirda, E.: Scalable, behavior-based malware clustering. In: NDSS. vol. 9, pp. 8-11 (2009)

3. Broder, A.Z.: On the resemblance and containment of documents. In: Compression and Complexity of Sequences 1997. Proceedings. pp. 21-29. IEEE (1997)

4. Chen, K., Liu, P., Zhang, Y.: Achieving accuracy and scalability simultaneously in detecting application clones on android markets. In: Proceedings of the 36th International Conference on Software Engineering. pp. 175-186. ACM (2014)

5. Chen, K., Wang, P., Lee, Y., Wang, X., Zhang, N., Huang, H., Zou, W., Liu, P.: Finding unknown malice in 10 seconds: Mass vetting for new threats at the google-play scale. In: USENIX Security Symposium. vol. 15 (2015)

6. Crussell, J., Gibler, C., Chen, H.: Attack of the clones: Detecting cloned applications on android markets. In: Computer Security-ESORICS 2012, pp. 37-54. Springer (2012)

7. Dexdump. http://developer.android.com/tools/help/index.html (2015)

8. Egele, M., Brumley, D., Fratantonio, Y., Kruegel, C.: An empirical study of cryptographic misuse in android applications. In: Proceedings of the 2013 ACM SIGSAC conference on Computer \& communications security. pp. 73-84. ACM (2013)

9. Glenn Fowler, Landon Curt Noll, K.P.V.: Fnv hash. http://www.isthe.com/ chongo/tech/comp/fnv/ (2015)

10. Grace, M., Zhou, Y., Zhang, Q., Zou, S., Jiang, X.: Riskranker: scalable and accurate zero-day android malware detection. In: Proceedings of the 10th international conference on Mobile systems, applications, and services. pp. 281-294. ACM (2012)

11. Hanna, S., Huang, L., Wu, E., Li, S., Chen, C., Song, D.: Juxtapp: A scalable system for detecting code reuse among android applications. In: Detection of Intrusions and Malware, and Vulnerability Assessment, pp. 62-81. Springer (2013)

12. Hu, X., Shin, K.G.: DUET: integration of dynamic and static analyses for malware clustering with cluster ensembles. In: Annual Computer Security Applications Conference (2013)

13. Hu, X., Shin, K.G., Bhatkar, S., Griffin, K.: Mutantx-s: Scalable malware clustering based on static features. In: USENIX Annual Technical Conference. pp. 187-198 (2013) 
14. Jang, J., Brumley, D., Venkataraman, S.: Bitshred: feature hashing malware for scalable triage and semantic analysis. In: Proceedings of the 18th ACM conference on Computer and communications security. pp. 309-320. ACM (2011)

15. Kang, M.G., Poosankam, P., Yin, H.: Renovo: A hidden code extractor for packed executables. In: Proceedings of the 2007 ACM workshop on Recurring malcode. pp. 46-53. ACM (2007)

16. Kim, J., Krishnapuram, R., Davé, R.: Application of the least trimmed squares technique to prototype-based clustering. Pattern Recognition Letters 17(6), 633641 (1996)

17. Korczynski, D.: ClusTheDroid: Clustering Android Malware. Master's thesis, Royal Holloway University of London (2015)

18. Leskovec, J., Rajaraman, A., Ullman, J.D.: Mining of massive datasets. Cambridge University Press (2014)

19. Li, Y., Sundaramurthy, S.C., Bardas, A.G., Ou, X., Caragea, D., Hu, X., Jang, J.: Experimental study of fuzzy hashing in malware clustering analysis. In: Proceedings of the 8th USENIX Conference on Cyber Security Experimentation and Test. pp. 8-8. USENIX Association (2015)

20. Martignoni, L., Christodorescu, M., Jha, S.: Omniunpack: Fast, generic, and safe unpacking of malware. In: Computer Security Applications Conference, 2007. ACSAC 2007. Twenty-Third Annual. pp. 431-441. IEEE (2007)

21. Meng, G., Xue, Y., Xu, Z., Liu, Y., Zhang, J., Narayanan, A.: Semantic modelling of android malware for effective malware comprehension, detection, and classification. In: Proceedings of the 25th International Symposium on Software Testing and Analysis. pp. 306-317. ACM (2016)

22. Rieck, K., Trinius, P., Willems, C., Holz, T.: Automatic analysis of malware behavior using machine learning. Journal of Computer Security 19(4), 639-668 (2011)

23. Roberts, J.: VirusShare.com. http://virusshare.com/ (2015)

24. Royal, P., Halpin, M., Dagon, D., Edmonds, R., Lee, W.: Polyunpack: Automating the hidden-code extraction of unpack-executing malware. In: Proceedings of the 22nd Annual Computer Security Applications Conference. pp. 289-300. IEEE Computer Society (2006)

25. Samra, A.A.A., Yim, K., Ghanem, O.A.: Analysis of clustering technique in android malware detection. In: Innovative Mobile and Internet Services in Ubiquitous Computing (IMIS), 2013 Seventh International Conference on. pp. 729-733. IEEE (2013)

26. Santos, I., Nieves, J., Bringas, P.G.: Semi-supervised learning for unknown malware detection. In: International Symposium on Distributed Computing and Artificial Intelligence. pp. 415-422. Springer (2011)

27. Sebastián, M., Rivera, R., Kotzias, P., Caballero, J.: Avclass: A tool for massive malware labeling. In: International Symposium on Research in Attacks, Intrusions, and Defenses. pp. 230-253. Springer (2016)

28. Snell, B.: Mobile threat report, what's on the horizon for 2016. http:// www.mcafee.com/us/resources/reports/rp-mobile-threat-report-2016.pdf (2016)

29. Virustotal. https://www.virustotal.com (2017)

30. Ye, Y., Li, T., Chen, Y., Jiang, Q.: Automatic malware categorization using cluster ensemble. In: ACM SIGKDD International Conference on Knowledge Discovery and Data mining (2010)

31. Zhang, F., Huang, H., Zhu, S., Wu, D., Liu, P.: Viewdroid: towards obfuscationresilient mobile application repackaging detection. In: Proceedings of the 2014 
Yuping Li, Jiyong Jang, Xin $\mathrm{Hu}$, and Xinming $\mathrm{Ou}$

ACM conference on Security and privacy in wireless \& mobile networks. pp. 25-36. $\mathrm{ACM}(2014)$

32. Zheng, M., Sun, M., Lui, J.: DroidAnalytics: A signature based analytic system to collect, extract, analyze and associate Android malware. In: Trust, Security and Privacy in Computing and Communications (TrustCom), 2013 12th IEEE International Conference on. pp. 163-171. IEEE (2013)

33. Zhou, W., Zhou, Y., Jiang, X., Ning, P.: Detecting repackaged smartphone applications in third-party Android marketplaces. In: Proceedings of the second ACM conference on Data and Application Security and Privacy. pp. 317-326. ACM (2012)

34. Zhou, Y., Jiang, X.: Dissecting android malware: Characterization and evolution. In: Security and Privacy (SP), 2012 IEEE Symposium on. pp. 95-109. IEEE (2012)

\section{A Detailed malicious payload mining results}

Table 3. Malicious payload under popular libraries

\begin{tabular}{|c|c|}
\hline Family & Popular Class Names Used \\
\hline Nickispy & $\begin{array}{l}\text { com.google.android.info.SmsInfo } \\
\text { com.google.android.service.UploadService }\end{array}$ \\
\hline Uuserv & $\begin{array}{l}\text { com.uuservice.status.SysCaller.callSilentInstall } \\
\text { com.uuservice.status.SilenceTool.MyThread.run }\end{array}$ \\
\hline Fjcon & $\begin{array}{l}\text { com.android.XWLauncher.CustomShirtcutActivity } \\
\text { com.android.XWLauncher.InstallShortcutReceiver }\end{array}$ \\
\hline Yzhc & $\begin{array}{c}\text { com.android.Base.Tools.replace_name } \\
\text { com.android.JawbreakerSuper.Deamon }\end{array}$ \\
\hline Gumen & $\begin{array}{l}\text { com.umeng.adutils.AdsConnect } \\
\text { com.umeng.adutils.SplashActivity }\end{array}$ \\
\hline Basebridge & $\begin{array}{l}\text { com.android.sf.dna.Collection } \\
\text { com.android.battery.a.pa }\end{array}$ \\
\hline Spambot & $\begin{array}{l}\text { com.android.providers.message.SMSObserver } \\
\text { com.android.providers.message.Utils.sendSms }\end{array}$ \\
\hline Moavt & $\begin{array}{l}\text { com.android.MJSrceen.Activity.BigImageActivity } \\
\text { com.android.service.MouaService.InitSms }\end{array}$ \\
\hline Zitmo & $\begin{array}{l}\text { com.android.security.SecurityService.onStart } \\
\text { com.android.smon.SecurityReceiver.sendSMS }\end{array}$ \\
\hline Mseg & $\begin{array}{c}\text { com.google.vending.CmdReceiver } \\
\text { android.ad.appoffer.Copy_2_of_DownloadManager }\end{array}$ \\
\hline Droidkungfu & $\begin{array}{l}\text { com.google.ssearch.SearchService } \\
\text { com.google.update.UpdateService }\end{array}$ \\
\hline Dowgin & $\begin{array}{c}\text { com.android.qiushui.app.dmc } \\
\text { com.android.game.xiaoqiang.jokes.Data9 }\end{array}$ \\
\hline Fakeinst & $\begin{array}{c}\text { com.googleapi.cover.Actor } \\
\text { com.android.shine.MainActivity.proglayss_Click }\end{array}$ \\
\hline Ksapp & $\begin{array}{c}\text { com.google.ads.analytics.Googleplay } \\
\text { com.google.ads.analytics.ZipDecryptInputStream }\end{array}$ \\
\hline Bankun & $\begin{array}{c}\text { com.google.game.store.bean.MyConfig.getMsg } \\
\text { com.google.dubest.eight.isAvilible }\end{array}$ \\
\hline Pjapps & $\begin{array}{l}\text { com.android.MainService.SMSReceiver } \\
\text { com.android.main.TANCActivity }\end{array}$ \\
\hline Adwo & $\begin{array}{c}\text { com.android.mmreader1030 } \\
\text { com.google.ads.AdRequest.isTestDevice }\end{array}$ \\
\hline Svpeng & $\begin{array}{c}\text { com.adobe.flashplayer_.FV.doInBackground } \\
\text { com.adobe.flashplayer_.FA.startService }\end{array}$ \\
\hline Opfake & $\begin{array}{l}\text { com.android.appupdate.UpdateService } \\
\text { com.android.system.SurpriseService }\end{array}$ \\
\hline Badao & $\begin{array}{l}\text { com.google.android.gmses.MyApp } \\
\text { com.android.secphone.FileUtil.clearTxt }\end{array}$ \\
\hline
\end{tabular}

